\title{
Randomized controlled trial for improved recovery of the pelvic floor after vaginal delivery with a specially formulated postpartum supplement
}

\author{
Peter Takacs, MD, PhD, MBA ${ }^{1}$, Bence Kozma, MD, PhD², Rudolf Lampé, MD, PhD², Attila Sipos, MD, ${ }^{2}$ \\ Robert Poka, MD, PhD² \\ 'Division of Female Pelvic Medicine and Reconstructive Surgery, Department of Obstetrics and Gynecology, Eastern Virginia Medical School, Norfolk, \\ VA, USA; ${ }^{2}$ Department of Obstetrics and Gynecology, University of Debrecen Faculty of Medicine, Debrecen, Hungary
}

\section{Objective}

To improve pelvic floor recovery after vaginal delivery with daily supplementation of a specially formulated postpartum recovery supplement.

\section{Methods}

Within 48 hours of vaginal delivery, primipara women were randomized in a 1:1 ratio to receive daily oral supplementation for 6 weeks with either a combination of regular prenatal vitamin (PNV), leucine (4 g/day), zinc (30 mg/day) and omega-3 fatty acid (900 mg/day) (treatment group), or only a PNV daily (control group). Co-primary outcomes were vaginal squeeze pressure as measured by perineometer and levator muscle injury as measured by transperineal 3-dimensional tomographic ultrasound at 6 weeks postpartum.

\section{Results}

Twenty-six women in the control group and 27 in the treatment group completed the trial. Weak pelvic floor muscle strength was significantly less frequent in the treatment group compared to the control group at 6 weeks after delivery ( $28 \%$ vs. $58 \%, P=0.03)$. Both right and left-sided levator-urethra gap was significantly larger in the control group compared to the treatment group indicating more levator injury being present in the control group at 6 weeks after delivery. Anterior vaginal wall prolapse at or beyond the hymenal ring was significantly more common in the control group compared to the treatment group ( $19 \%$ vs. $0 \%, P=0.02)$. Significantly more women reported bothersome bulge symptoms in the control group compared to the treatment group at 6 weeks postpartum (19\% vs. $0 \%, P=0.02)$.

\section{Conclusion}

Postpartum women who received a specially formulated postpartum recovery supplement had improved recovery of the pelvic floor after vaginal delivery.

Keywords: Postpartum; Pelvic floor; Omega-3 fatty acids; Zinc; Leucine

\section{Introduction}

Approximately $25 \%$ of women suffer from a degree of incontinence, prolapse, or vaginal laxity due to the weakening of the pelvic floor after a "normal" delivery $[1,2]$. There is an association between urinary incontinence, pelvic floor muscle strength, and the number and type of deliveries $[3,4]$. During a vaginal delivery, it is quite common $(15-50 \%$ of women
Received: 2019.09.29. Revised: 2019.11.07. Accepted: 2019.11.26. Corresponding author: Peter Takacs, MD, PhD, MBA

Division of Female Pelvic Medicine and Reconstructive Surgery, Department of Obstetrics and Gynecology, Eastern Virginia Medical School, 825 Fairfax Avenue, Suite 526, Norfolk, VA 23507 2007, USA

E-mail: takacsp@evms.edu

https://orcid.org/0000-0002-0078-5763

Articles published in Obstet Gynecol Sci are open-access, distributed under the terms of the Creative Commons Attribution Non-Commercial License (http://creativecommons. org/licenses/by-nc/3.0/) which permits unrestricted non-commercial use, distribution, and reproduction in any medium, provided the original work is properly cited.

Copyright $\odot 2020$ Korean Society of Obstetrics and Gynecology 


\section{Obstetrics \& Gynecology Science}

Vol. 63, No. 3, 2020

may be affected) for the pelvic floor muscles (levator) to be damaged to some extent [5-7]. If this condition is not adequately repaired and persists for an extended period of time, it can lead to increased risk for pelvic floor dysfunction. After delivery, more than $60 \%$ of patients recover from pelvic floor muscle injuries within 1 year [8]. However, women with persistent levator avulsion show significantly worse deterioration patterns in muscle strength, hiatus measurements, and vaginal symptoms such as a loose vagina or lump sensation $[8,9]$. These findings emphasize the importance of proper recovery from pelvic floor muscle injuries sustained at birth. Currently, we are not aware of any preventive methods; such methods could significantly reduce injuries to the pelvic floor muscles during pregnancy and childbirth.

Zinc is the second most common trace element in the human body and is a cofactor for many enzymes [10]. It plays a vital role in connective tissue biosynthesis, and we have investigated the role of zinc in previous publications $[11,12]$. We have shown that in human vaginal smooth muscle cells, $20 \mu \mathrm{M}$ zinc levels have a beneficial effect on the production of extracellular components produced by the muscle, which thereby increase the levels of collagen and elastin that are produced, as well as the amount of smooth muscle. In addition, animal experiments have demonstrated the importance of zinc $[13,14]$. One animal study showed that zinc plays an important role in vaginal extracellular matrix (ECM) composition: plasma zinc levels were significantly lower in buffaloes with antepartum prolapse [14]. Additionally, when rats were kept on a diet lacking zinc, the structure of the vagina became similar to a menopausal vagina's structure [13]. Another study found that when zinc replacement was performed locally in the vagina, the vaginal ECM regenerated, and a vaginal ECM resembling that of juvenile rats was created [11]. In humans, tissue zinc levels are significantly lower in patients with all types of hernias, and risk factors for the development of hernias are similar to those for the development of vaginal prolapse [15]. Even if there is no absolute zinc deficiency in pregnant women, the risk of peripartum and postpartum complications is still increased by zinc serum levels that are at the lower end of the normal range [16-18]. While maternal zinc levels did not differ before delivery, plasma zinc levels were significantly decreased in women who underwent vaginal deliveries, relative to the zinc levels of women who received cesarean sections [16]. This is probably due to the high stress placed on skeletal and uterine muscles during childbirth [16].

The beneficial effects of omega-3 fatty acids in pregnancy are well known. It has been shown that omega-3 fatty acids can increase the production of muscle proteins, thereby enhancing muscle strength [19-22]. Dietary intake of omega-3 fatty acids increases muscle anabolic signaling activity, thereby stimulating protein synthesis in muscles [20].

Leucine is an essential amino acid that is also capable of stimulating the synthesis of muscle proteins. It enhances regeneration and prevents muscle tissue from degradation. Leucine can also prevent muscular weakness related to aging [23]. Numerous studies have documented leucine's beneficial role in enhancing muscle protein synthesis [23-25].

The aim of this study was to investigate the recovery of pelvic floor injuries and their related symptoms after daily supplementation with a specially formulated dietary supplement. This supplement was designed to promote the restoration of muscular injuries following delivery and contains ingredients of a multivitamin composition that can be taken during lactation. The key ingredients of the supplement were zinc, leucine, and omega-3 fatty acids.

\section{Materials and methods}

We conducted a randomized, double-blind, placebo-controlled trial with a 6-week follow-up period. A 1:1 randomization ratio was generated and completed by SAS (SAS Institute, Cary, NC, USA) version 9.4. We enrolled postpartum women who underwent a normal prenatal course without significant medical comorbidities, and who had a first-time, normal vaginal delivery in the Department of Obstetrics and Gynecology at the University of Debrecen in Hungary. Women were enrolled within 48 hours of delivery, after signing a written informed-consent form. Exclusion criteria were as follows: one or more previous pregnancies with vaginal delivery (multipara), multiple gestations, premature birth, operative vaginal delivery (other than outlet vacuum delivery), gestational diabetes, caesarean section, allergy to fish, vegetarian lifestyle, history of uterine malformation or prior uterine surgery. Furthermore, patients with known zinc or copper deficiencies and patients with collagen or connective tissue diseases were also excluded.

After enrollment, women were randomized to receive the specially formulated dietary supplement (Mommytoo vita- 


\section{Obstetrics \& Gynecology Science}

Peter Takacs, et al. Postpartum supplement: a randomized trial

min; Fempharma, LLC, Budapest, Hungary) (treatment group) or a prenatal vitamin (PNV; control group). Women in the treatment group were asked to take the oral dietary supplement every day for 6 weeks. The dietary supplement in the treatment group included a daily dose of PNV, $4 \mathrm{~g}$ of leucine, $30 \mathrm{mg}$ of zinc, and $900 \mathrm{mg}$ omega-3 fatty acids (465 mg eicosapentaenoic acid and $375 \mathrm{mg}$ docosahexaenoic acid). The daily supplementation in control group women consisted of a routine PNV.

After randomization, subjects received the dietary supplement in a sealed, uniform box. The completely identical boxes were labeled with codes that were not known by researchers participating in the clinical trial. The patients could not be identified by the code. Participants did not know what kind of preparations they were given (i.e., what the tablets looked like in the 2 groups), and were randomly assigned to a group without informing them to which group they belonged. After enrollment, women were asked to complete the Pelvic Organ Prolapse Distress Inventory 6 (POPDI-6) and the Urinary Distress Inventory 6 (UDI-6). Serum samples were obtained to measure zinc levels in a certified laboratory. At the 6 week post-delivery visit, a general gynecological exam was performed and a POP-Q measurement was obtained. In addition, the strength of the voluntary contractions of the vaginal muscles were measured by a perineometer (Peritron; Laborie, Williston, VT, USA) in a standardized fashion $[26,27]$.
A 3-dimensional (3D) pelvic floor ultrasound was also performed to evaluate defects in the musculus levator ani using GE Voluson S8 3D ultrasound equipment (GE Medical Systems, Chicago, IL, USA). Both the levator hiatus and the levator-urethra gap (LUG) was measured as described previously [28-30]. Serum sample was taken from the participants to determine their serum zinc and copper level. Participants were asked to complete the POPDI-6 and UDI-6 questionnaires again.

Co-primary outcomes were the measurement of vaginal squeeze force by a perineometer and the measurement of musculus levator ani defects by ultrasound (LUG) [26-31].

Secondary outcomes were questionnaire (POPD- 6 and UDI6) results, Pelvic Organ Prolapse Quantification (POP-Q) measurements, a digital survey of pelvic floor muscle strength, and serum zinc concentrations. These were performed immediately following delivery and at 6 weeks after delivery.

\section{Questionnaires}

The POPDI- 6 and UDI- 6 were used to assess subjective pelvic floor symptoms [32]. To specify the severity of pelvic floor distress symptoms, patient response options ranged from 0 ("no symptoms") to 4 ("quite a bit"). To calculate the score, the mean score of answered items within each component was multiplied by 25 to obtain the scale score (range $0-100$ ).

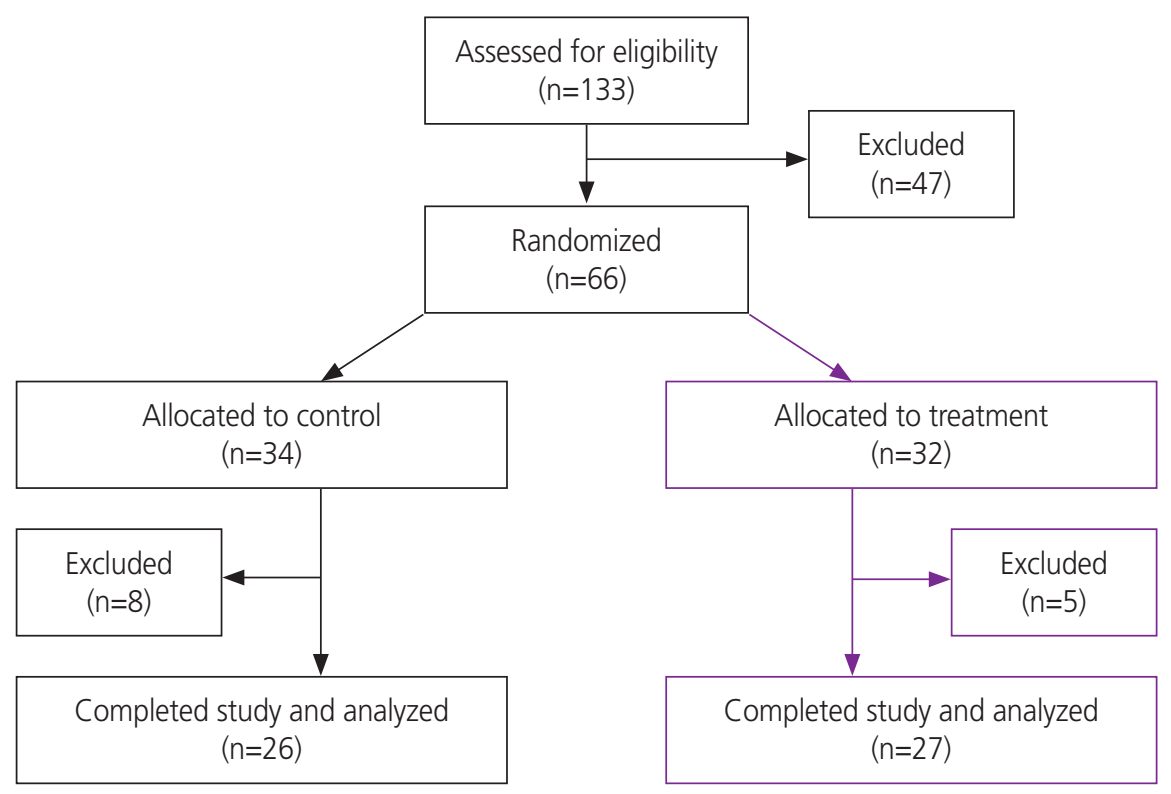

Fig. 1. Flow of participants through the randomized clinical trial. Participants were allocated to treatment or control groups. 


\title{
Obstetrics \& Gynecology Science
}

\author{
Vol. 63 , No. 3, 2020
}

\section{Statistics}

For statistical analyses, we used SigmaStat/SPSS software. Descriptive statistics were calculated for all variables of interest. Means and standard deviations (SDs) were calculated for continuous outcomes. Frequency and percentage were calculated for categorical outcomes. Student's t-test was used to compare the mean values between the 2 groups. Paired $t$-test was used to compare paired data, which were obtained at baseline and at 6 weeks (zinc levels, questionnaires). Frequencies were analyzed by Fisher's exact test. This study had adequate power to detect a difference after 46 patients' enrollment. Power analysis was performed based on our pilot study, which revealed that the threshold for a weak pelvic floor muscle is $\leq 17 \mathrm{~cm} \mathrm{H}_{2} \mathrm{O}$ as measured by our perineometer. To have a power of $80 \%$ and a significance level of $5 \%$ to detect a $40 \%$ difference in the rate of weak vaginal squeeze pressure, a sample size of 46 patients was required, with 23 patients in each arm of our randomized clinical trial (RCT). Statistical significance was defined as a $P$-value $<0.05$ using 2-tailed tests.

\section{Results}

Fig. 1 summarizes the assessment for eligibility, randomization, allocation, and exclusions of participants. Of the 113 women screened, 66 met entry criteria and were randomized to treatment or control groups. Fifty-three women completed the trial. Of the 47 participants who were excluded, 10 did not meet inclusion criteria, and 37 decided not to participate in the trial. Thirty-four women were randomly assigned to the control group and 32 to the treatment group.

Of the 32 individuals randomly assigned to treatment group, 5 did not complete the trial: 2 were lost to followup, 3 withdrew from participation because of minor adverse events ( 1 woman developed a rash, 1 woman could not swallow pills, and 1 mother felt that her infant's vomiting was related to the supplement). Of the 34 women randomized to the control group, 8 did not complete the trial: 4 were lost to follow-up, 2 withdrew consent before taking any supplements, 1 developed a minor adverse event (rash), and 1 refused any examination at the follow-up visit. In total, 53 women completed the trial: 26 in the control group and 27 in the treatment group.

\section{Demographic and baseline characteristics at randomization}

Demographic and baseline characteristics at randomization are presented in Table 1. There were no statistically significant differences in the distribution of any of the demographic variables between the control and treatment groups. The majority of women had a mediolateral episiotomy at delivery. Birthweight and fetal head circumference were similar between the control and treatment group.

Table 1. Demographic and clinical characteristics of participants

\begin{tabular}{|c|c|c|c|}
\hline Characteristics & Control group $(n=26)$ & Treatment group $(n=27)$ & $P$-value \\
\hline Age (yr) & $28 \pm 3$ & $29 \pm 3$ & NS \\
\hline Gravida & $1(1-3)$ & $1(1-3)$ & NS \\
\hline Pre-pregnancy BMI (kg/m²) & $21.7 \pm 4.6$ & $21.9 \pm 3.8$ & NS \\
\hline BMI at delivery $\left(\mathrm{kg} / \mathrm{m}^{2}\right)$ & $28.3 \pm 4.3$ & $26.9 \pm 3.3$ & NS \\
\hline Estimated gestational age (wk) & $38.6 \pm 1.4$ & $38.7 \pm 1.4$ & NS \\
\hline Birthweight (grams) & $3,307 \pm 401$ & $3,411 \pm 111$ & NS \\
\hline Head circumference (cm) & $33 \pm 1$ & $33 \pm 1$ & NS \\
\hline Length of second stage (min) & $27 \pm 11$ & $32 \pm 13$ & NS \\
\hline Episiotomy & $25(96)$ & $25(92)$ & NS \\
\hline Epidural & $18(69)$ & $16(60)$ & NS \\
\hline Vacuum extraction & $0(0)$ & $3(11)$ & NS \\
\hline Breastfeeding & $26(100)$ & $27(100)$ & NS \\
\hline
\end{tabular}

Values are presented as mean \pm standard deviation, median (range) or number (\%).

BMI, body mass index; NS, not significant. 


\section{Obstetrics \& Gynecology Science}

Peter Takacs, et al. Postpartum supplement: a randomized trial

\section{Serum zinc levels}

We expected that zinc levels would rise in the treatment and control groups compared to the baseline values obtained within 48 hours after delivery, since both the control and treatment group received daily zinc supplementation (15 mg vs. $30 \mathrm{mg}$, respectively). Consistent with the patient-reported compliance in both the treatment and control groups, the mean zinc levels increased significantly from baseline compared to 6 weeks after delivery (Table 2). Although the mean zinc level was 10\% higher in the treatment group at 6 weeks postpartum compared to the control group, it did not reach statistical significance. However, zinc levels on average increased significantly more in the treatment group from base- line to 6 weeks postpartum compared to the control group (mean \pm SD $\Delta$ zinc level increase $0.48 \pm 0.24$ vs. $0.29 \pm 0.20$, $P<0.01)$.

\section{Co-primary outcomes}

1) Vaginal squeeze pressure as measured by perineometer at 6 weeks after delivery

The results obtained by the perineometer are described in Table 3. The mean Oxford scale values were similar between groups. However, weak pelvic floor muscle strength (defined as the mean of 3 vaginal squeeze pressure measurements by Peritron perineometer $\leq 17 \mathrm{~cm} \mathrm{H}_{2} \mathrm{O}$ ) was significantly less fre-

Table 2. Serum zinc levels at baseline and 6 weeks postpartum

\begin{tabular}{lccc}
\hline Characteristics & Control group $(\mathbf{n}=\mathbf{2 6})$ & Treatment group $(\mathbf{n}=\mathbf{2 7})$ & $\boldsymbol{P}$-value \\
\hline Zinc level after delivery $(\mathrm{mg} / \mathrm{L})$ & $0.71 \pm 0.19$ & $0.62 \pm 0.10$ & $\mathrm{NS}$ \\
Zinc level 6 weeks postpartum $(\mathrm{mg} / \mathrm{L})$ & $1.00 \pm 0.19$ & $1.10 \pm 0.25$ & $\mathrm{NS}$ \\
P-value & $<0.01$ & $<0.01$ & - \\
\hline
\end{tabular}

Values are presented as mean \pm standard deviation.

NS, not significant.

Table 3. Measurement of vaginal squeeze pressure by perineometer at 6 weeks postpartum and manual assessment of pelvic floor strength on a pelvic exam by palpation (Oxford scale)

\begin{tabular}{|c|c|c|c|c|}
\hline Characteristics & $\begin{array}{l}\text { Control group } \\
(n=26)\end{array}$ & $\begin{array}{l}\text { Treatment group } \\
\qquad(n=27)\end{array}$ & $\begin{array}{c}\text { Risk ratio } \\
\text { (95\% confidence interval) }\end{array}$ & $P$-value \\
\hline Vaginal squeeze pressure $\left(\mathrm{cm} \mathrm{H}_{2} \mathrm{O}\right)$ & $21 \pm 17$ & $22 \pm 13$ & N/A & NS \\
\hline Oxford scale & $3(1-5)$ & $3(1-4)$ & N/A & NS \\
\hline Weak vaginal squeeze pressure ( $\left.\leq 17 \mathrm{~cm} \mathrm{H}_{2} \mathrm{O} ; \%\right)$ & 58 & 28 & $0.48(0.23-0.98)$ & 0.03 \\
\hline
\end{tabular}

Values are presented as mean \pm standard deviation or median (range).

NS, not significant; N/A, not applicable.

Table 4. Levator-urethra gap (LUG) and levator hiatus measured by 3-dimensional transperineal tomographic ultrasound at 6 weeks postpartum

\begin{tabular}{|c|c|c|c|c|}
\hline Characteristics & $\begin{array}{l}\text { Control group } \\
\qquad(n=26)\end{array}$ & $\begin{array}{l}\text { Treatment group } \\
\qquad(\mathrm{n}=27)\end{array}$ & $\begin{array}{c}\text { Risk ratio } \\
(95 \% \text { confidence interval) }\end{array}$ & $P$-value \\
\hline Right-sided LUG (mm) & $24.9 \pm 4.2$ & $21.1 \pm 2.9$ & $\mathrm{~N} / \mathrm{A}$ & $<0.01$ \\
\hline Left-sided LUG (mm) & $24.9 \pm 3.7$ & $20.1 \pm 2.8$ & $\mathrm{~N} / \mathrm{A}$ & $<0.01$ \\
\hline Levator hiatus $\left(\mathrm{cm}^{2}\right)$ & $17.7 \pm 2.7$ & $15.5 \pm 2.4$ & $\mathrm{~N} / \mathrm{A}$ & $<0.01$ \\
\hline Unilateral avulsion (\%) & 34.6 & 3.7 & $0.10(0.01-0.78)$ & $<0.01$ \\
\hline Bilateral avulsion (\%) & 38.4 & 3.7 & $0.09(0.01-0.70)$ & $<0.01$ \\
\hline
\end{tabular}

Values are presented as mean \pm standard deviation.

N/A, not applicable. 


\title{
Obstetrics \& Gynecology Science
}

\author{
Vol. 63, No. 3, 2020
}

quent in the treatment group compared to the control group at 6 weeks post-delivery ( $28 \%$ vs. $58 \%, P=0.03$ ). The risk of having a weak vaginal squeeze pressure was reduced by $52 \%$ in the treatment group compared to the control group at 6 weeks postpartum (Risk ratio [RR], 0.48; 95\% confidence interval $[\mathrm{Cl}], 0.23-0.98 ; P=0.03)$.

2) Levator-urethra gap as measured by 3-dimensional transperineal tomographic ultrasound

Both right- and left-sided LUGs were significantly larger in the control group compared to the treatment group (Table 4). A large LUG $(\geq 25 \mathrm{~mm})$ is a good indicator of a levator muscle injury and typically indicates avulsion. Unilateral levator injury was defined by an LUG $\geq 25 \mathrm{~mm}$ on at least one side. Unilateral levator avulsion was significantly less frequent in the treatment group compared to the control group at 6 weeks after delivery ( $3.7 \%$ vs. $34.6 \%, P<0.01)$. The presence of unilateral levator avulsion was reduced by almost $90 \%$ compared to the control group at 6 weeks postpartum (RR, 0.10; $95 \% \mathrm{Cl}, 0.01-0.78)$.

Bilateral avulsion was defined by LUG $\geq 25 \mathrm{~mm}$ on both sides. Similar to unilateral avulsion, bilateral levator avulsion was significantly less frequent in the treatment group compared to the control group at 6 weeks after delivery $(3.7 \%$ vs. $38.4 \%, P<0.01)$. The presence of bilateral levator avulsion was reduced by $90 \%$ compared to the control group at 6 weeks postpartum (RR, 0.09; $95 \% \mathrm{Cl}, 0.01-0.70 ; P<0.01)$. In addition, the levator hiatus was significantly smaller in the treatment group compared to the control group at 6 weeks

Table 5. Pelvic Organ Prolapse Quantification (POP-Q) measurement of women at 6 weeks postpartum

\begin{tabular}{lccc}
\hline Characteristics & $\begin{array}{c}\text { Control } \\
\text { group }(\mathbf{n = 2 6})\end{array}$ & $\begin{array}{c}\text { Treatment } \\
\text { group }(\mathbf{n}=\mathbf{2 7})\end{array}$ & P-value \\
\hline Aa & $-1.40 \pm 1.00$ & $-1.63 \pm 0.80$ & NS \\
Ba & $-1.40 \pm 1.00$ & $-1.63 \pm 0.80$ & NS \\
C & $-6.00 \pm 0.80$ & $-6.20 \pm 0.90$ & NS \\
GH & $3.56 \pm 0.70$ & $3.40 \pm 0.70$ & NS \\
PB & $2.67 \pm 0.50$ & $2.56 \pm 0.40$ & NS \\
TVL & $9.80 \pm 0.70$ & $9.80 \pm 0.80$ & NS \\
Ap & $-1.73 \pm 0.90$ & $-1.70 \pm 0.90$ & NS \\
Bp & $-1.73 \pm 0.90$ & $-1.70 \pm 0.90$ & NS \\
D & $-7.50 \pm 3.20$ & $-7.80 \pm 0.94$ & NS \\
\hline
\end{tabular}

NS, not significant. after delivery $(15.5 \pm 2.4$ vs. $17.7 \pm 2.7, P<0.01)$.

\section{Secondary outcomes}

1) Pelvic Organ Prolapse Quantification measurement The findings of the POP-Q measurement are described in Table 5. Anterior vaginal wall prolapse at or beyond the hymenal ring was significantly more common in the control group compared to the treatment group (19\% vs. $0 \%$, $P=0.02$ ). Also, wide genital hiatus (defined as $\mathrm{GH} \geq 4 \mathrm{~cm}$ ) was significantly more frequent in the control group compared to the treatment group at 6 weeks after delivery ( $46 \%$ vs. $18 \%, P=0.01)$. The risk of wide genital hiatus was almost 3 times higher in the control group compared to the treatment group 6 weeks postpartum (RR, 2.95; $95 \% \mathrm{Cl}, 1.19$ 7.30; $P=0.01)$.

2) Validated questionnaires (Pelvic Organ Prolapse Distress Inventory 6, Urinary Distress Inventory 6) The mean POPDI- 6 and UDI- 6 standardized scale scores were similar between the control and treatment groups $(6.6 \pm 13.0$ vs. $4.6 \pm 6.3, P=0.46$ and $18.4 \pm 16.8$ vs. $22.3 \pm 14.5, P=0.60$ ). However, significantly more women reported bothersome bulge symptoms (Q\#3 on POPDI-6) in the control group compared to the treatment group at 6 weeks postpartum (19\% vs. $0 \%, P=0.02$ ). In addition, the mean standardized POPDI-6 score significantly improved in the treatment group at 6 weeks compared to the baseline score $(4.6 \pm 6.3$ vs. $1.7 \pm 3.9$, $P=0.02$ ) but did not improve significantly in the control group $(6.6 \pm 13.0$ vs. $2.2 \pm 3.5, P=0.11)$. The mean standardized UDI6 score significantly improved in both the control and the treatment groups by 6 weeks compared to the baseline score $(18.4 \pm 16.8$ vs. $3.8 \pm 5.5, P<0.01$ [control] and $22.3 \pm 14.5$ vs. 5.4 $\pm 9.7, P<0.01$ [treatment group]).

\section{Discussion}

We are the first to report the effects of a specially formulated postpartum recovery supplement on the recovery of pelvic floor function after vaginal delivery. We found that daily supplementation resulted in improved recovery of the pelvic floor after a normal vaginal delivery. Previous publications have showed that after delivery, pelvic floor muscle injuries recover within 1 year, in more than $60 \%$ of cases [8]. Howev- 


\section{Obstetrics \& Gynecology Science}

Peter Takacs, et al. Postpartum supplement: a randomized trial

er, women with persistent levator avulsion show significantly worse deterioration patterns in muscle strength, hiatus measurements, and vaginal symptoms (e.g., loose vagina or lump sensations) $[8,9]$. These findings emphasize the importance of proper recovery from pelvic floor muscle injuries sustained at delivery.

Pelvic floor muscle injuries have been linked to anterior and central compartment prolapse and likely represent the missing link between delivery and the development of prolapse. A levator avulsion seems to increase the risk of significant anterior and central compartment prolapse by 3-fold [33]. Our findings are in agreement with prior observations. We have found that anterior vaginal wall prolapse at or beyond the hymenal ring was significantly more common in the control group compared to the treatment group. This may be explained by the fact that we found that significantly larger LUG in the control group was an indicator of more levator avulsion, which caused more anterior vaginal wall prolapse. In addition, a wide genital hiatus (defined as $\mathrm{GH} \geq 4 \mathrm{~cm}$ ) was significantly more frequent in the control group compared to the treatment group at 6 weeks after delivery also likely the result of the presence of levator injuries. Similar to the presence of significant anterior vaginal wall prolapse, bothersome prolapse symptoms were more common in the control group.

Vaginal squeeze pressure has been previously used as a marker for pelvic floor function [34]. Levator injury has been associated with weaker pelvic floor muscle contraction measured with palpation, perineometry, and ultrasound [35]. Weak vaginal squeeze pressure has been associated with more anterior compartment prolapse and with minor and major avulsion [9]. Weak pelvic floor muscle strength (defined as the mean of 3 vaginal squeeze pressure measurements by Peritron perineometer $\leq 17 \mathrm{~cm} \mathrm{H}_{2} \mathrm{O}$ ) was significantly less frequent in our treatment group compared to the control group at 6 weeks after delivery, likely secondary to less levator injury being present.

We believe that our specially formulated postpartum supplement contributed to the faster and superior recovery of the pelvic floor, secondary to the special composition of the supplement. Zinc serum levels have been shown to significantly decrease after vaginal delivery, but not after elective cesarean sections [16]. Our supplement contained higher doses of zinc compared to the control group. Despite no difference in the mean serum zinc levels between the control and treatment group at 6 weeks, zinc levels on average increased significantly more in the treatment group from baseline to 6 weeks postpartum compared to the control group. In addition, our supplement contained high doses of the essential amino acid leucine. Leucine is the only nutritional amino acid capable of stimulating the synthesis of muscle proteins [24]. It is directly involved in protein synthesis as it directly stimulates protein synthesis in muscles, but it has not been shown to contribute to pelvic floor recovery [23-25]. The beneficial effect of omega-3 fatty acids in pregnancy is well known. In humans, it has been shown that omega-3 fatty acids can increase the production of muscle proteins, thereby enhancing muscle strength [19-21].

Our findings indicate that the special composition of our supplement containing all 3 key ingredients (zinc, leucine and omega-3 fatty acid) in a unique ratio may be responsible for the observed beneficial effects on pelvic floor recovery. The composition of the supplement was based on the tolerable upper intake level (UL) of zinc, which is $40 \mathrm{mg}$. The UL represents the maximum daily intake that is unlikely to cause adverse health effects. For this reason, women took $30 \mathrm{mg}$ of zinc as a supplement to the normal dietary intake, but not exceeding the UL of $40 \mathrm{mg}$ per day. Leucine was tested previously in doses up to $10 \mathrm{gm}$ per day and found to be safe [23-25]. One of the main problems with leucine supplementation is the large amounts required. Our aim was to identify the smallest dose possible that was still effective for our postpartum women. Previously, it was shown that omega-3 fatty acids can increase the production of muscle proteins and that $900 \mathrm{mg}$ is a safe well-established dose. In addition, omega-3 fatty acids have been tried in clinical trials at doses up to $4 \mathrm{gm}$ a day [19-22].

The strength of this study was its randomized nature and that it is the first trial to test the effects of a food supplement on the recovery of postpartum pelvic floor function. An important strength of the study was that study participants were blinded to the allocation. Another strength of this $\mathrm{RCT}$ is that the investigators performing the perinoemetry, the transperineal ultrasound, and the pelvic floor assessment were also blinded to the allocation. In addition, the measurements of LUG were performed prior to breaking the randomization. Also, a strength of this study was our participants' high compliance in taking the supplements, as reflected by the significant elevation of zinc serum levels in both the treatment and control groups. Previous research has 


\section{Obstetrics \& Gynecology Science}

Vol. 63, No. 3, 2020

revealed that after vaginal delivery, zinc levels are expected to decrease significantly. However, in our study population, zinc levels increased significantly, suggesting patient compliance with the allocated supplements [16]. The trial discontinuation rate in the treatment group was not statistically different compared to the control group ( $15.6 \%$ vs. $23.5 \%, P>0.05$ ). The up to $80 \%$ attrition rate is comparable to that of other randomized controlled trials.

The main limitations of our study include the lack of capability to detect differences in several secondary outcomes and the relatively low number of women enrolled. Another was that we did not assess levator injury either by ultrasound or by perineometry immediately after delivery. In our experience, it is quite difficult to accurately assess levator injuries immediately after delivery, secondary to the tissue swelling, edema, and pain that may be involved with the examinations. A further weakness of our study was the homogenous non-diverse study population, which can be explained by the fact that the vast majority of the local population belongs to only one race and ethnicity. Another limitation to the generalizability of this study is the fact that over $90 \%$ of women had an episiotomy during delivery. Our trial did not provide accurate insight into the mechanism of action of the supplement. It is possible that the observed effects are secondary through an anti-inflammatory process rather than a direct effect on the levator muscles and ECM.

In summary, patients receiving the specially formulated postpartum recovery supplement showed improved recovery in pelvic floor function after vaginal delivery. Postpartum women who received the supplement were less likely to have weak vaginal squeeze pressure, presence of levator muscle injury, anterior vaginal prolapse at or beyond the hymenal ring, bothersome bulge symptoms, and wide genital hiatus compared to women who received a PNV only at 6 weeks after vaginal delivery. Further research is needed to validate our findings in a more diverse and larger population.

\section{Acknowledgements}

This work was supported by GINOP-2.1.1-15-2016-00783 (Economic Development and Innovation Operational Program Grant of the European Union and Hungary).

\section{Conflict of interest}

Peter Takacs is a paid consultant for Fempharma LLC. The other authors have no potential conflict of interest relevant to this article was reported.

\section{Ethical approval}

The trial was approved and registered by the Hungarian National Institutional Review Medical Research Council \#84483/2018/EUIG.

\section{Patient consent}

The patients provided written informed consent for the publication.

\section{References}

1. Rørtveit G, Hannestad YS. Association between mode of delivery and pelvic floor dysfunction. Tidsskr Nor Laegeforen 2014;134:1848-52.

2. Chaliha C. Postpartum pelvic floor trauma. Curr Opin Obstet Gynecol 2009;21:474-9.

3. Özdemı ÖÇ, Bakar Y, Özengın N, Duran B. The effect of parity on pelvic floor muscle strength and quality of life in women with urinary incontinence: a cross sectional study. J Phys Ther Sci 2015;27:2133-7.

4. Hilde G, Stær-Jensen J, Siafarikas F, Engh ME, Brækken $I H, B \varnothing K$. Impact of childbirth and mode of delivery on vaginal resting pressure and on pelvic floor muscle strength and endurance. Am J Obstet Gynecol 2013;208:50.e1-7.

5. Dietz HP, Simpson JM. Does delayed child-bearing increase the risk of levator injury in labour? Aust N Z J Obstet Gynaecol 2007;47:491-5.

6. Dietz HP. Ultrasound in the assessment of pelvic organ prolapse. Best Pract Res Clin Obstet Gynaecol 2019;54:12-30.

7. Atan IK, Lin S, Dietz HP, Herbison P, Wilson PD; ProLong Study Group. Levator avulsion is associated with pelvic organ prolapse 23 years after the first childbirth. J Ultra- 


\section{Obstetrics \& Gynecology Science}

Peter Takacs, et al. Postpartum supplement: a randomized trial

sound Med 2018;37:2829-39.

8. van Delft KW, Thakar R, Sultan AH, IntHout J, Kluivers KB. The natural history of levator avulsion one year following childbirth: a prospective study. BJOG 2015;122:1266-73.

9. van Delft K, Sultan AH, Thakar R, Schwertner-Tiepelmann $\mathrm{N}$, Kluivers $\mathrm{K}$. The relationship between postpartum levator ani muscle avulsion and signs and symptoms of pelvic floor dysfunction. BJOG 2014;121:1164-71.

10. National Institutes of Health (US). Zinc: fact sheet for health professionals. Bethesda (MD): National Institutes of Health; 2020 [cited 2019 Sep 28]. Available from: https://ods.od.nih.gov/factsheets/Zinc-HealthProfessional/.

11. Takacs P, Jaramillo $S$, Zhang Y, Datar R, Williams A, OIczyk J, et al. The effects of PPAR $\delta$ agonist and zinc on ovariectomized rats' vagina. Female Pelvic Med Reconstr Surg 2013;19:126-31.

12. Takacs P, Zhang Y, Candiotti K, Jaramillo S, Medina CA. Effects of PPAR-delta agonist and zinc on vaginal smooth muscle cells collagen and tropoelastin production. Int Urogynecol J Pelvic Floor Dysfunct 2012;23:1775-9.

13. Taneja SK, Kaur R. Pathology of ovary, uterus, vagina and gonadotrophs of female mice fed on $\mathrm{Zn}$-deficient diet. Indian J Exp Biol 1990;28:1058-65.

14. Kelkar MA, Khar SK, Mandakhot VM. Studies on antepartum prolapse of the vagina in buffalo--plasma trace element concentrations. Arch Exp Veterinarmed 1989;43:315-8.

15. Ozdemir S, Ozis ES, Gulpinar K, Aydin SM, Eren AA, Demirtas $S$, et al. The value of copper and zinc levels in hernia formation. Eur J Clin Invest 2011;41:285-90.

16. Schulpis KH, Karakonstantakis T, Vlachos GD, Mentis $A F$, Karikas GA, Afordakou $D$, et al. Maternal-neonatal magnesium and zinc serum concentrations after vaginal delivery. Scand J Clin Lab Invest 2010;70:465-9.

17. Lazebnik N, Kuhnert BR, Kuhnert PM, Thompson KL. Zinc status, pregnancy complications, and labor abnormalities. Am J Obstet Gynecol 1988;158:161-6.

18. Caulfield LE, Zavaleta N, Shankar AH, Merialdi M. Potential contribution of maternal zinc supplementation during pregnancy to maternal and child survival. Am J Clin Nutr 1998;68:499S-508S.

19. Smith GI, Julliand S, Reeds DN, Sinacore DR, Klein S, Mittendorfer B. Fish oil-derived n-3 PUFA therapy increases muscle mass and function in healthy older adults. Am J
Clin Nutr 2015;102:115-22.

20. Smith GI, Atherton P, Reeds DN, Mohammed BS, Rankin D, Rennie MJ, et al. Dietary omega-3 fatty acid supplementation increases the rate of muscle protein synthesis in older adults: a randomized controlled trial. Am J Clin Nutr 2011;93:402-12.

21. Gingras AA, White PJ, Chouinard PY, Julien P, Davis TA, Dombrowski L, et al. Long-chain omega-3 fatty acids regulate bovine whole-body protein metabolism by promoting muscle insulin signalling to the Akt-mTOR-S6K1 pathway and insulin sensitivity. J Physiol 2007;579:269-84.

22. Di Girolamo FG, Situlin R, Mazzucco S, Valentini R, Toigo G, Biolo G. Omega-3 fatty acids and protein metabolism: enhancement of anabolic interventions for sarcopenia. Curr Opin Clin Nutr Metab Care 2014;17:145-50.

23. Trabal J, Forga M, Leyes P, Torres F, Rubio J, Prieto E, et al. Effects of free leucine supplementation and resistance training on muscle strength and functional status in older adults: a randomized controlled trial. Clin Interv Aging 2015;10:713-23.

24. Rowlands DS, Nelson AR, Phillips SM, Faulkner JA, Clarke J, Burd NA, et al. Protein-leucine fed dose effects on muscle protein synthesis after endurance exercise. Med Sci Sports Exerc 2015;47:547-55.

25. Luiking YC, Deutz NE, Memelink RG, Verlaan S, Wolfe RR. Postprandial muscle protein synthesis is higher after a high whey protein, leucine-enriched supplement than after a dairy-like product in healthy older people: a randomized controlled trial. Nutr J 2014;13:9.

26. Afshari P, Dabagh F, Iravani M, Abedi P. Comparison of pelvic floor muscle strength in nulliparous women and those with normal vaginal delivery and cesarean section. Int Urogynecol J Pelvic Floor Dysfunct 2017;28:1171-5.

27. Myer EN, Roem JL, Lovejoy DA, Abernethy MG, Blomquist JL, Handa VL. Longitudinal changes in pelvic floor muscle strength among parous women. Am J Obstet Gynecol 2018;219:482.e1-7.

28. Dietz HP, Abbu A, Shek KL. The levator-urethra gap measurement: a more objective means of determining levator avulsion? Ultrasound Obstet Gynecol 2008;32:941-5.

29. Dietz HP, Garnham AP, Rojas RG. Is the levator-urethra gap helpful for diagnosing avulsion? Int Urogynecol J Pelvic Floor Dysfunct 2016;27:909-13.

30. Kozma B, Larson K, Scott L, Cunningham TD, Abuhamad $A$, Poka $R$, et al. Association between pelvic organ 


\section{Obstetrics \& Gynecology Science}

Vol. 63, No. 3, 2020

prolapse types and levator-urethra gap as measured by 3D transperineal ultrasound. J Ultrasound Med 2018;37:2849-54.

31. Kamisan Atan I, Shek KL, Furtado Gl, Caudwell-Hall J, Dietz HP. The association between levator-urethra gap measurements and symptoms and signs of female pelvic organ prolapse. Female Pelvic Med Reconstr Surg 2016;22:442-6.

32. Barber MD, Walters MD, Bump RC. Short forms of two condition-specific quality-of-life questionnaires for women with pelvic floor disorders (PFDI-20 and PFIQ-7). Am J
Obstet Gynecol 2005;193:103-13.

33. Dietz HP, Simpson JM. Levator trauma is associated with pelvic organ prolapse. BJOG 2008;115:979-84.

34. Pereira VS, Hirakawa HS, Oliveira AB, Driusso P. Relationship among vaginal palpation, vaginal squeeze pressure, electromyographic and ultrasonographic variables of female pelvic floor muscles. Braz J Phys Ther 2014;18:428-34.

35. Nyhus MØ, Salvesen KÅ, Volløyhaug I. Association between pelvic floor muscle trauma and contraction in parous women from a general population. Ultrasound Obstet Gynecol 2019;53:262-8. 